Article

\title{
Investigating the Systems-Level Effect of Pueraria lobata for Menopause-Related Metabolic Diseases Using an Ovariectomized Rat Model and Network Pharmacological Analysis
}

\author{
Ji Hong Oh ${ }^{1,+}$, Seon-Eun Baek ${ }^{2,+}$, Won-Yung Lee ${ }^{1}{ }^{\mathbb{C}}$, Ji Yun Baek ${ }^{3,4}{ }^{\oplus}$, Tuy An Trinh ${ }^{3}$, \\ Do Hwi Park ${ }^{3}$, Hye Lim Lee ${ }^{5}$, Ki Sung Kang ${ }^{3}$, Chang-Eop Kim ${ }^{1} *$ (D) and Jeong-Eun Yoo ${ }^{2, *(D)}$ \\ 1 Department of Physiology, College of Korean Medicine, Gachon University, Seongnam 13120, Korea; \\ jihong421@hanmail.net (J.H.O.); wonyung21@naver.com (W.-Y.L.) \\ 2 Department of Obstetrics and Gynecology, College of Korean Medicine, Daejeon University, \\ Daejeon 35235, Korea; dreamingmong@naver.com \\ 3 Department of Preventive Medicine, College of Korean Medicine, Gachon University, \\ Seongnam 13120, Korea; wldbsttn@naver.com (J.Y.B.); tuyantrinh@gmail.com (T.A.T.); \\ parkdo@gachon.ac.kr (D.H.P.); kkang@gachon.ac.kr (K.S.K.) \\ 4 Department of Food Science, Gyeongnam National University of Science and Technology, Jinju 52725, Korea \\ 5 Department of Pediatrics, College of Korean Medicine, Daejeon University, Daejeon 35235, Korea; \\ hanilim@dju.ac.kr \\ * Correspondence: eopchang@gachon.ac.kr (C.-E.K.); jeyoo@dju.ac.kr (J.-E.Y.); Tel.: +82-31-750-5493 (C.-E.K.); \\ +82-42-470-9139 (J.-E.Y.) \\ + These authors contributed equally to this work.
}

Received: 6 September 2019; Accepted: 14 November 2019; Published: 18 November 2019

check for updates

\begin{abstract}
This study was conducted to evaluate the biological activities of Pueraria lobata (PL) on menopause-related metabolic diseases and to explore the underlying mechanism of PL by network pharmacological analyses. We used ovariectomized (OVX) rats as a postmenopausal model and administered PL at different doses (50,100, and $200 \mathrm{mg} / \mathrm{kg}$ ). In OVX rats, decreased uterine weights and PPAR- $\gamma$ (peroxisome proliferator-activated receptor-gamma) mRNA expression in the thigh muscle were significantly recovered after PL administration. PL also significantly alleviated OVX-induced increases in total cholesterol, triglyceride, alanine aminotransferase (ALT/GPT), and aspartate aminotransferase (AST/GOT) levels. To identify the systems-level mechanism of PL, we performed network pharmacological analyses by predicting the targets of the potential bioactive compounds and their associated pathways. We identified 61 targets from four potential active compounds of PL: formononetin, beta-sitosterol, 3'-methoxydaidzein, and daidzein-4,7-diglucoside. Pathway enrichment analysis revealed that among female sex hormone-related pathways, the estrogen signaling pathways, progesterone-mediated oocyte maturation, oxytocin signaling pathways, and prolactin signaling pathways were associated with multiple targets of PL. In conclusion, we found that PL improved various indicators associated with lipid metabolism in the postmenopausal animal model, and we also identified that its therapeutic effects are exerted via multiple female sex hormone-related pathways.
\end{abstract}

Keywords: menopause-related metabolic diseases; menopause; Pueraria lobata; network pharmacology; lipid metabolism; dyslipidemia 


\section{Introduction}

Menopause is a period when women's ovarian function gradually declines and female hormones fluctuate and decrease, resulting in irregular menstruation and menopause-related symptoms. Lack of estrogen induces imbalance of energy homeostasis, by assisting energy intake and lipogenesis, depressing energy expenditure, and aggravating insulin secretion and sensitivity [1]. These metabolic changes in menopause mediate the development of chronic diseases such as cardiovascular diseases (CVD), obesity, hyperlipidemia, and fatty liver [2]. The prevalence of the metabolic syndrome in postmenopausal women is higher than in premenopausal women, which is estimated at $31-55 \%$ according to the various studies [3-6]. In postmenopausal life, prevention and management of these chronic diseases is critical for maintaining good health.

Hormone replacement therapy (HRT) is used as the first choice for treating different menopausal disabling symptoms. While HRT is effective in alleviating most postmenopausal symptoms [7], many women refuse HRT concerning the latent adverse effects and prefer the use of nonhormonal therapies to relieve symptoms after menopause [7,8]. As one of the nonhormonal treatments, natural products are considered as an alternative to menopausal symptoms and metabolic diseases caused by menopause. In particular, herbs have been widely used to relieve symptoms after menopause in East Asia, especially in Korea, China, and Japan $[9,10]$. Herb-based therapies were found to be associated with more reductions in the symptoms in menopausal women than in the placebo group in meta-analysis [11]. In a randomized controlled trial, whole soy had a beneficial effect on cardiovascular biomarkers in equol-producing postmenopausal women when compared with the placebo group [12]. Several experimental studies reported that traditional herbal prescriptions reduced the serum lipid levels and hepatic lipid accumulation in an ovariectomized rat model $[13,14]$.

Pueraria lobata (PL), the essential herb in traditional Korean medicine, is used for the treatment of various diseases such as diabetes, liver diseases, CVD, and postmenopausal osteoporosis [15-18]. PL extracts have also been reported to prevent obesity and improve glucose metabolism [19]. Pueraria lobata is expected to function as an alternative to estrogen because it contains isoflavones-for instance, puerarin, daidzein, daidzin, genistin, and genistein—which are representative phytoestrogens that show structural or functional similarity to estrogen [20,21].

Network pharmacology, the novel approach based on systems biology, enables the elucidation of the potential mechanism of multiple compounds and the identification of pathways associated with the targets of the compounds at the systems level [22]. This approach can also be applied to the study of diseases affecting various systems of the body (e.g., menopause-related metabolic diseases). Since PL is composed of multiple compounds, including phytoestrogens, this would be a proper method to investigate the mechanism of PL in menopause-related metabolic diseases.

Here, we aimed to investigate the potential benefits of PL on menopause-related metabolic diseases at the systems level. To evaluate the biological activity of PL, we assessed changes in uterine weight, serum lipids, alanine aminotransferase (ALT/GPT), aspartate aminotransferase (AST/GOT), liver weight, and peroxisome proliferator-activated receptor gamma (PPAR- $\gamma$ ) messenger RNA (mRNA) expression in an ovariectomized (OVX) rat model. To explore the underlying mechanism of PL, we investigated target genes and pathways of PL related to menopause-related metabolic diseases by applying network pharmacology analysis (Figure 1). 


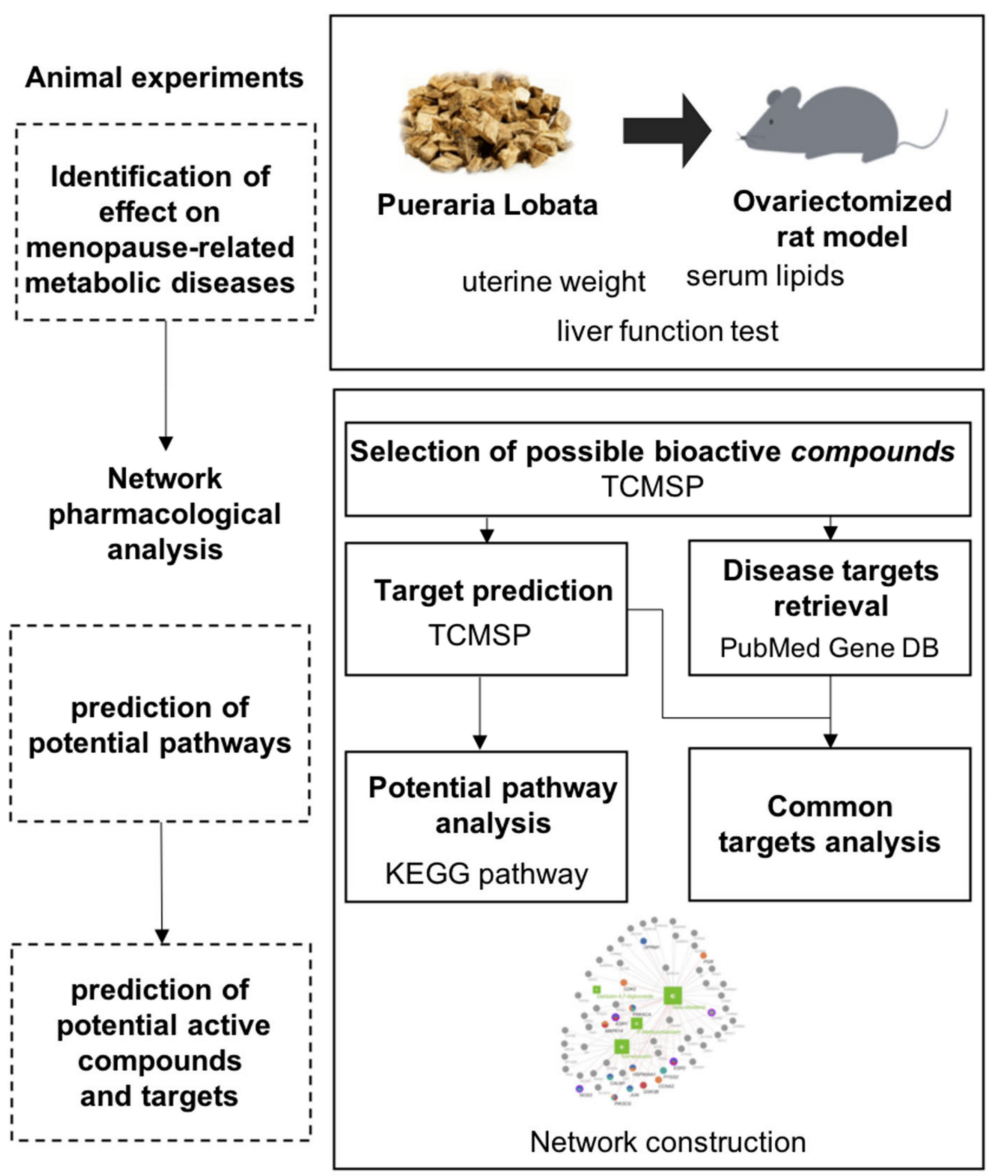

Figure 1. Overview of the study process. Abbreviations: TCMSP, Traditional Chinese Medicine Systems Pharmacology; KEGG, Kyoto Encyclopedia Genes and Genomes.

\section{Materials and Methods}

\subsection{Preparation of the Herbal Formulation}

The PL (harvested from Gyeongnam, Geochang, Republic of Korea) was purchased from Okchundang Herbs (Seoul, Korea). Pueraria lobata (500 g) was extracted with $80 \% \mathrm{MeOH}(1.5 \mathrm{~L})$ three times at $25^{\circ} \mathrm{C}$, and $103.3 \mathrm{~g}$ of PL extract was obtained after drying under reduced pressure. The powdery dry extract of clomidin tablets (purchased from Seoul Pharmaceutical Co., Seoul, Republic of Korea), after the film coating composed of the dried extract of red clover (RC) was removed, was used as a positive control.

\subsection{Experimental Animals and Experimental Design}

We followed the Guidelines for Animal Experimentation approved by Gachon University in this study (GIACUC 2017-034). The experiment was conducted on 46 female Sprague-Dawley rats, five weeks old and weighing 130-150 g (Doo Yeol Biotech, Seoul, Korea). Housing conditions were maintained at $24 \pm 2{ }^{\circ} \mathrm{C}$; relative humidity was between $50 \%$ and $55 \%$. Food and water were available ad libitum. After one week of acclimatization, all rats were anesthetized with an intraperitoneal injection of Zoletil ${ }^{\circledR}$ (Virbac, Carros, France) and Rompun ${ }^{\circledR}$ (TS, Bayer, Leverkusen, Germany) mixture (3:1, $0.2 \mathrm{mg} / \mathrm{kg}$ body weight). Sham operation was performed for six rats (sham), and bilateral ovariectomies were performed for the remaining 40 rats. After surgery, rats were fed a solid diet for one week of recovery. Ovariectomized rats were divided into five groups $(n=8)$ : (1) OVX; (2) OVX + RC; (3) OVX + PL50; (4) OVX + PL100; and (5) OVX + PL200. The animals of the OVX + RC group were orally administered $3.3 \mathrm{mg} / \mathrm{kg}$ red clover extract. The animals in the OVX + PL50, OVX + PL100, 
and OVX + PL200 groups were orally treated with PL in aqueous solution at 50, 100, and $200 \mathrm{mg} / \mathrm{kg}$ bw, respectively. The animals in the sham and OVX groups were administered the same volume of distilled water for eight weeks. To investigate the effect of PL on menopause-related metabolic diseases, we collected blood, uterine, liver, and thigh muscle samples from each animal model.

\subsection{Blood and Organ Dissection}

At the end of the experimental period, the blood samples were collected via cardiac puncture. The uterus and liver were excised from the experimental animals and washed with saline, and the excess moisture was removed and weighed. The thigh muscles were dissected and stored in the freezer for the measurement of PPAR- $\gamma$ mRNA expression.

\subsection{Serum Analyses}

Blood samples were collected in test tubes containing $0.18 \mathrm{M}$ ethylenediaminetetraacetic acid (EDTA) and centrifuged at 3,500 revolution per minute (rpm) for $10 \mathrm{~min}$ at $4{ }^{\circ} \mathrm{C}$. The total cholesterol (range: $3.86-800 \mathrm{mg} / \mathrm{dL}$ ), high-density lipoprotein (HDL) cholesterol (range: 3-120 mg/dL), triglyceride (detection range: $8.85-885 \mathrm{mg} / \mathrm{dL}$ ), AST (detection range: $5-700 \mathrm{U} / \mathrm{L}$ ), and ALT (detection range: 5-700 U/L) were analyzed using the appropriate enzymatic colorimetric kits in accordance with the manufacturer's instructions (Roche, Mannheim, Germany). The serum levels of total cholesterol (Catalog No. 05168538190, Roche), HDL (Catalog No. 05168805190, Roche), triglycerides (Catalog No. 05171407 190, Roche), AST (Catalog No. 05850819190, Roche), and ALT (Catalog No. 05850797190, Roche) were measured using a kit (Roche).

\subsection{Measurement of PPAR- $\gamma$ mRNA Expression}

\subsubsection{Tissues and Total RNA Extraction}

The thigh muscles were separated, placed in a nitrogen tank for rapid freezing, and then stored at $-80^{\circ} \mathrm{C}$ until use. The homogenized muscle tissue was mixed with $200 \mu \mathrm{L}$ of chloroform (Sigma Co, St. Louis, MO, USA) per $1 \mathrm{~mL}$ of TRIzol solution (Favorgen Biotech Corp., Kaohsiung, Taiwan) for RNA extraction and centrifuged $\left(13,000 \mathrm{rpm}, 10 \mathrm{~min}, 4^{\circ} \mathrm{C}\right)$ to collect RNA from the supernatant.

\subsection{2. cDNA Preparation}

The RNA, DEPC (diethyl pyrocarbonate), and reverse transcriptase and Oligo (dT) 15 were mixed in tubes for polymerase chain reaction (PCR). Thereafter, the reaction was carried out at $75^{\circ} \mathrm{C}$ for $5 \mathrm{~min}$ using a PCR machine and a cDNA synthesis kit (Intron Biotechnology, Seoul, Korea). Then, the reaction mixture was reacted at $75^{\circ} \mathrm{C}$ for $5 \mathrm{~min}$, and $50 \mu \mathrm{L}$ of DEPC water was added to the obtained cDNA to be used for real time polymerase chain reaction (RT-PCR).

\subsubsection{Real-Time Polymerase Chain Reaction}

The synthesized cDNA $(1 \mu \mathrm{L})$ and the PPAR- $\gamma$ primer $(2 \mu \mathrm{L})$ were put into a master mix solution (Intron biotechnology), and $17 \mu \mathrm{L}$ of DEPC water was added, followed by amplification by PCR. The PCR products were then electrophoresed on $2 \%$ agarose gels, stained with ethidium bromide, and checked with an ultraviolet (UV) lamp (NEOScience, Seoul, Korea). After PCR, the amount of PPAR $-\gamma$ was calculated using an image station (Kodak, Rochester, USA) apparatus (Table 1).

Table 1. Primer sequence used for polymerase chain reaction (PCR).

\begin{tabular}{ccc}
\hline Primer & Sense $\left(5^{\prime}-3^{\prime}\right)$ & Antisense $\left(5^{\prime}-\mathbf{3}^{\prime}\right)$ \\
\hline $\begin{array}{c}\text { Peroxisome proliferator-activated } \\
\text { receptor gamma (PPAR- } \gamma)\end{array}$ & TCPLAPLGCTCTGTCATC & CATCTGTACTPLTPLPLACA \\
\hline
\end{tabular}




\subsection{Network Pharmacological Analysis}

The PL compounds were retrieved from the Traditional Chinese Medicine Systems Pharmacology (TCMSP, http://tcmspw.com/tcmsp.php) database [23]. Potential bioactive compounds were selected from compounds with an oral bioavailability $(\mathrm{OB}) \geq 30$ and drug-likeness (DL) index $\geq 0.18$, which were the default values suggested in the TCMSP [24]. We predicted potential bioactive compound targets from the TCMSP and further analyzed related signaling pathways of predicted targets using the Kyoto Encyclopedia Genes and Genomes (KEGG) database [25].

To understand the action of PL's bioactive compounds on menopausal symptoms, we retrieved menopause-related genes from the Entrez Gene database (ncbi.nlm.nih.gov/gene) [26]. Then, we identified common targets between predicted targets from the TCMSP and retrieved targets from the Entrez Gene database. After integrating information about potential bioactive compounds of PL, their targets, and related pathways, we constructed a network graph using Cytoscape 3.5.1 (http://cytoscape.org) [27].

\subsection{Statistical Processing}

Data were analyzed using the two-tailed Mann-Whitney $U$ test and the hypergeometric test. Statistical significance was set at the level of $p \leq 0.05$. Bonferroni correction was used for multiple comparisons. All statistical analyses were processed using the Scipy (http://scipy.org) and Numpy (http://Numpy.org) module in Python 3.6 (http://www.python.org).

\section{Results}

\subsection{Animal Experiments}

We used OVX rats as a postmenopausal model and administered PL at different doses (50, 100, and $200 \mathrm{mg} / \mathrm{kg}$ ).

\subsubsection{Uterine Weight}

The uterine weight in the OVX group showed a dramatic decrease compared to the sham group $(p=0.002$, Figure 2). It was observed that the PL treatment dose-dependently improved uterine weight in the OVX rats ( $p=0.008,0.0009$, and 0.0009 per dose, respectively), which is consistent with RC administration as a positive $(p=0.0009)$.

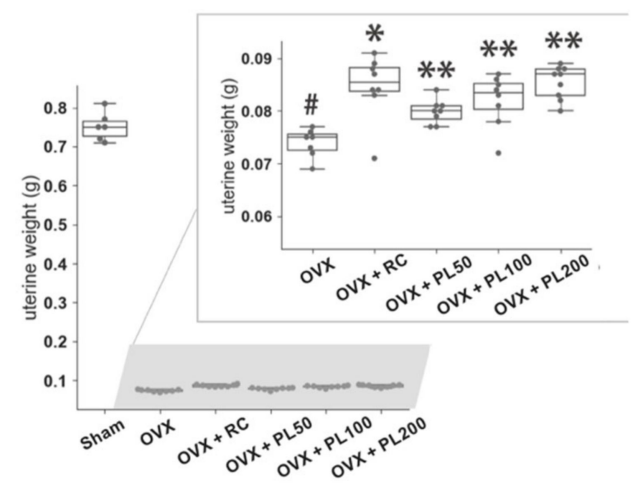

Figure 2. The effect of PL on the uterine weight of OVX rats. Zoomed graph is the data except for the sham group. Each point indicates the value of each animal. In the box plots, the upper and lower boundaries of the box mark the 75th percentile and the 25th percentile, respectively. A line within the box indicates the median, and whiskers above and below the box indicate 1.5 interquartile range (75th percentile-25th percentile). Points outside the box are identified as outliers. The results were compared by the Mann-Whitney U test; sham vs. OVX: $\# p \leq 0.05$; OVX vs. OVX + RC: ${ }^{*} \leq 0.05$; OVX vs. OVX + PL: **adjusted $p \leq 0.05$. 


\subsubsection{Serum Lipids}

We examined the levels of total cholesterol, triglyceride, and HDL (Figure 3). Prominent increases in serum lipids were noted in the OVX rats when compared with sham rats $(p=0.002$ for total cholesterol; $p=0.008$ for triglyceride; $p=0.008$ for HDL). Administration of $100 \mathrm{mg} / \mathrm{kg}$ PL resulted in a statistically significant reduction in total cholesterol $(p=0.007$, Figure $3 a)$ and triglyceride $(p=0.001$, Figure $3 \mathrm{~b}$ ), which were consistent with the positive control $(p=0.0007$ for total cholesterol; $p=0.0009$ for triglyceride). However, the levels of HDL showed no significant changes in all groups treated with PL (Figure 3c).

(a)

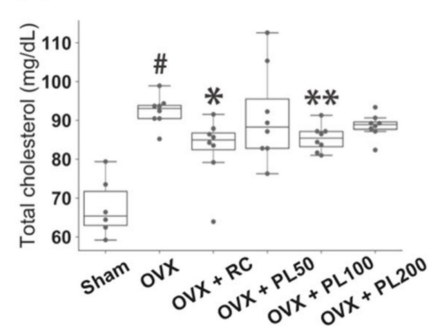

(b)

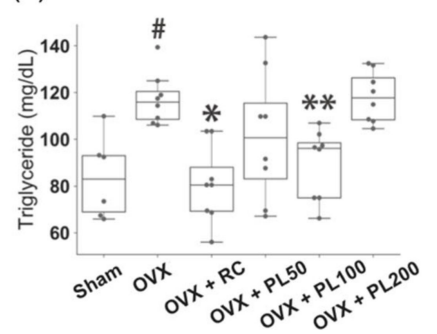

(c)

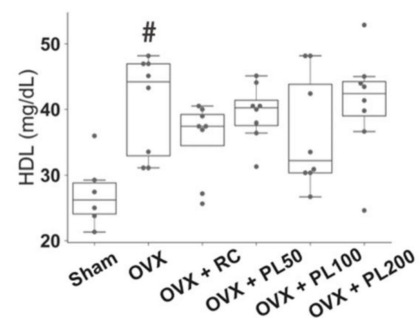

Figure 3. The effect of PL on serum lipids in OVX rats. (a) Total cholesterol; (b) triglyceride; (c) HDL; For description of the box plots, please refer to Figure 2. The results compared by the Mann-Whitney U test; sham vs. OVX: \# $p \leq 0.05$; OVX vs. OVX + RC: * $p \leq 0.05$; OVX vs. OVX + PL: **adjusted $p \leq 0.05$.

3.1.3. Glutamate Oxaloacetate Transaminase (GOT), Glutamate Pyruvate Transaminase (GPT), and Liver Weight

The GPT, GOT, and liver weights of OVX rats were markedly increased compared with those of sham rats ( $p=0.002$ for GPT; $p=0.03$ for GOT; $p=0.02$ for liver weight, Figure 4). After treatment with $100 \mathrm{mg} / \mathrm{kg}$ and $200 \mathrm{mg} / \mathrm{kg}$ PL, the GPT significantly reduced to levels similar to those in the sham $(p=0.002$ and 0.004 , respectively, Figure 4a). The administration of $50 \mathrm{mg} / \mathrm{kg}$ PL also reduced the GPT in the OVX + PL50, but the difference was not statistically significant with a Bonferroni correction $(p=0.03)$. The GOT also significantly decreased in the OVX + PL100 and OVX + PL200 ( $p=0.0009$ for both groups, Figure 4b). For the liver weight, we observed no significant changes in OVX + PL50, OVX + PL100, and OVX + PL200 with a Bonferroni correction $(p=0.02,0.02$, and 0.05 , respectively, Figure $4 c)$.

(a)

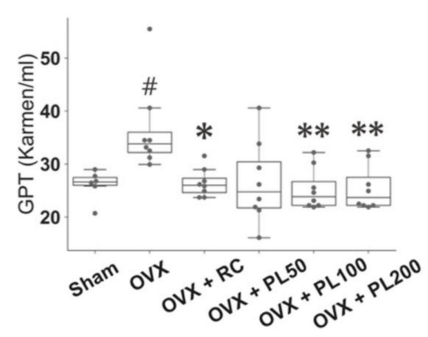

(b)

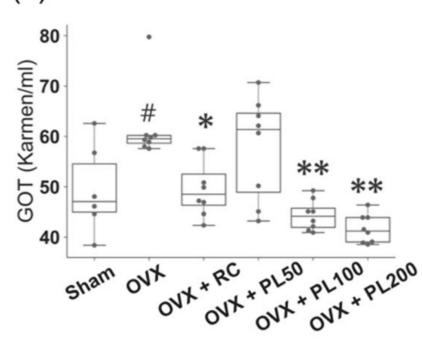

(c)

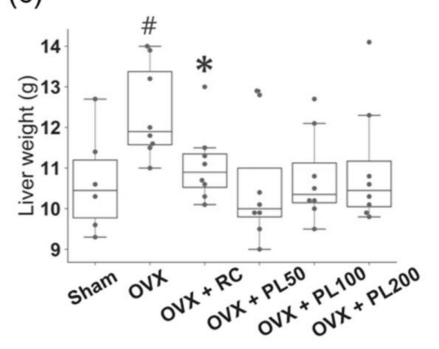

Figure 4. The effect of PL on liver function and liver weight of OVX rats. (a) GPT; (b) GOT; (c) liver weight. For description of the box plots, please refer to Figure 2. The results compared by the Mann-Whitney U test; sham vs. OVX: $\# p \leq 0.05$; OVX vs. OVX + RC: ${ }^{*} p \leq 0.05$; OVX vs. OVX + PL: ${ }^{* *}$ adjusted $p \leq 0.05$.

\subsubsection{Peroxisome Proliferator-Activated Receptor Gamma Messenger RNA Expression}

The PPAR- $\gamma$ mRNA expression in the thigh muscle decreased in the OVX group when compared with the sham $(p=0.002)$. The PPAR- $\gamma$ mRNA expression of OVX + PL50 $(p=0.006)$, OVX + PL100 $(p=0.001)$, and OVX + PL200 $(p=0.002)$ was significantly increased, similar to that of the OVX + RC group $(p=0.001)$ (Figure 5). 


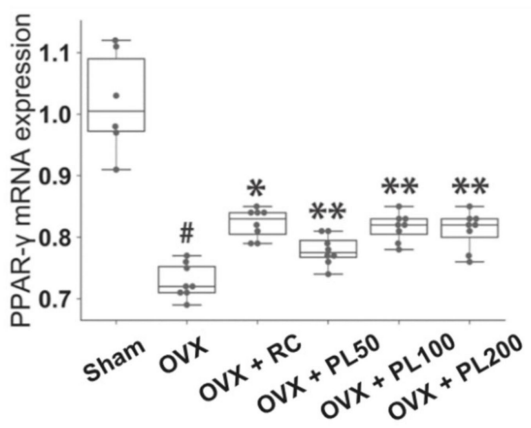

Figure 5. The effect of PL on PPAR- $\gamma$ mRNA expression in OVX rats. For description of the box plots, please refer to Figure 2. The results compared by the Mann-Whitney U test; sham vs. OVX: \#p $\leq 0.05$; OVX vs. OVX + RC; ${ }^{*} \leq \leq 0.05 ;$ OVX vs. OVX + PL: **adjusted $p \leq 0.05$.

\subsection{Network Pharmacological Analyses}

We performed network pharmacological analyses to determine PL's potential bioactive compounds and their targets and to predict the underlying mechanisms of PL on menopause-related metabolic diseases.

\subsubsection{Selection of Potential Bioactive Compounds of Pueraria lobata}

Eighteen PL compounds were retrieved from the TCMSP (Table 2). To evaluate the ADME (absorption, distribution, metabolism, and elimination or excretion), we considered the pharmacokinetic parameters of the compounds: oral bioavailability (OB) and drug likeness (DL). The OB represents the possibility of the drug reaching the target upon oral administration; the DL index evaluates whether a compound can be bioactive when compared to the known drugs. As a result, we identified four potential bioactive compounds satisfying the screening criteria ( $\mathrm{OB} \geq 30 \%, \mathrm{DL} \geq 0.18)$ : formononetin, beta-sitosterol, 3'-methoxydaidzein, and daidzein-4,7-diglucoside.

Table 2. Compounds of Pueraria lobata (PL) with their oral bioavailability (OB), drug likeness (DL), and molecular properties.

\begin{tabular}{|c|c|c|c|c|c|c|c|}
\hline Compound Name & MW & OB (\%) & DL & $A \log P$ & Hdon & Hacc & Caco-2 \\
\hline Formononetin * & 268.28 & 69.67 & 0.21 & 2.58 & 1 & 4 & 0.78 \\
\hline Sitogluside & 576.95 & 20.63 & 0.62 & 6.34 & 4 & 6 & -0.14 \\
\hline Beta-sitosterol ${ }^{*}$ & 414.79 & 36.91 & 0.75 & 8.08 & 1 & 1 & 1.32 \\
\hline Daidzein & 254.25 & 19.44 & 0.19 & 2.33 & 2 & 4 & 0.59 \\
\hline Ononin & 430.44 & 11.52 & 0.78 & 0.68 & 4 & 9 & -0.74 \\
\hline Docosanoate & 340.66 & 15.69 & 0.26 & 9.11 & 1 & 2 & 1.21 \\
\hline Lupenone & 424.78 & 11.66 & 0.78 & 7.36 & 0 & 1 & 1.48 \\
\hline Genistein & 270.25 & 17.93 & 0.21 & 2.07 & 3 & 5 & 0.43 \\
\hline Lignoceric acid & 368.72 & 14.9 & 0.33 & 10.02 & 1 & 2 & 1.24 \\
\hline Scoparone & 206.21 & 74.75 & 0.09 & 1.87 & 0 & 4 & 0.85 \\
\hline (R)-allantoin & 158.14 & 96.9 & 0.03 & -1.76 & 5 & 7 & -0.99 \\
\hline 3'-Methoxydaidzein * & 284.28 & 48.57 & 0.24 & 2.32 & 2 & 5 & 0.56 \\
\hline Daidzein-4,7-diglucoside ${ }^{*}$ & 578.57 & 47.27 & 0.67 & -1.48 & 8 & 14 & -2.53 \\
\hline Soyasapogenol b & 458.8 & 16.73 & 0.75 & 5.11 & 3 & 3 & 0.43 \\
\hline Puerarin & 416.41 & 24.03 & 0.69 & -0.06 & 6 & 9 & -1.15 \\
\hline $7,8,4^{\prime}$-Trihydroxyisoflavone & 270.25 & 20.67 & 0.22 & 2.07 & 3 & 5 & 0.45 \\
\hline Daidzin & 416.41 & 14.32 & 0.73 & 0.43 & 5 & 9 & -1 \\
\hline Sophoradiol & 442.8 & 17.42 & 0.76 & 6.2 & 2 & 2 & 0.95 \\
\hline
\end{tabular}

Note that compounds marked with an * satisfy the screening criteria (OB $\geq 30, \mathrm{DL} \geq 0.18)$. MW, molecular weight; AlogP, octanol-water partition coefficient $\log \mathrm{P}$; Hdon, hydrogen bond donor; Hacc, hydrogen bond acceptor; Caco-2, Caco-2 permeability. 


\subsubsection{Target Analysis}

With the potential bioactive compounds of PL, we identified 61 potential targets by using an in silico model from the TCMSP (Table 3). These targets are either experimentally validated or predicted by machine learning algorithms [28]. To identify the genes that are known to be associated with menopause among the 61 targets, we retrieved menopause-related genes from the Entrez Gene database. Four overlapping genes were found between the predicted 61 targets of PL's potential bioactive compounds and 110 menopause-related genes from the Entrez Gene database: estrogen receptor 1 (ESR1), estrogen receptor 2 (ESR2), nitric oxide synthase 3 (NOS3), and beta-2 adrenoreceptor (ADRB2).

Table 3. Predicted targets of the potential bioactive compounds of Pueraria lobata (61 genes).

\begin{tabular}{ccccccccc}
\hline \multicolumn{7}{c}{ Predicted Targets of the Potential Bioactive Compounds of PL } \\
\hline ESR1 & ESR2 & JUN & MT-ND6 & ATP5B & NOS2 & PTGS1 & F2 & CHRM1 \\
AR & PPARG & PTGS2 & NOS3 & CA2 & RXRA & ACHE & PDE3A & ADRA1A \\
PTPN1 & SLC6A3 & ADRB2 & SLC6A4 & DPP4 & MAPK14 & GSK3B & HSP90AA1 & CDK2 \\
ACPC & MAOB & CHEK1 & PRKACA & PRSS1 & PIM1 & CCNA2 & PKIA & CALM1 \\
BCL2 & PON1 & MAP2 & DRD1 & CHRM3 & KCNH2 & SCN5A & GABRA2 & CHRM4 \\
HTR2A & GABRA5 & GABRA3 & PGR & CHRM2 & ADRA1B & CHRNA2 & OPRM1 & NR3C1 \\
GABRA1 & PIK3CG & CHRNA7 & CAMC & NCOA2 & NCOA2 & TOP2A & & \\
\hline
\end{tabular}

\subsubsection{Identifying Potential Pathways of Pueraria lobata}

We performed KEGG pathway enrichment analysis to better understand the underlying pathways that are associated with the predicted 61 targets. A total of 107 enriched signaling pathways were obtained at an adjusted $p$-value of 0.05 , among which 30 pathways with high combined scores are presented in Table 4. We found that four pathways were related to the female sex hormones in the top 30 pathways: estrogen signaling pathway, progesterone-mediated oocyte maturation, oxytocin signaling pathway, and prolactin signaling pathway. Menopause-related genes (except $A D R B 2$ ) were also found to be involved in the female sex hormone-related pathways. These genes have been shown to be involved in an additional nine pathways that can be potential pathways by which PL relieves menopausal symptoms.

Table 4. KEGG pathway enrichment analysis of potential target genes of PL's potential bioactive compounds (adjusted $p$-value $\leq 0.05$ ).

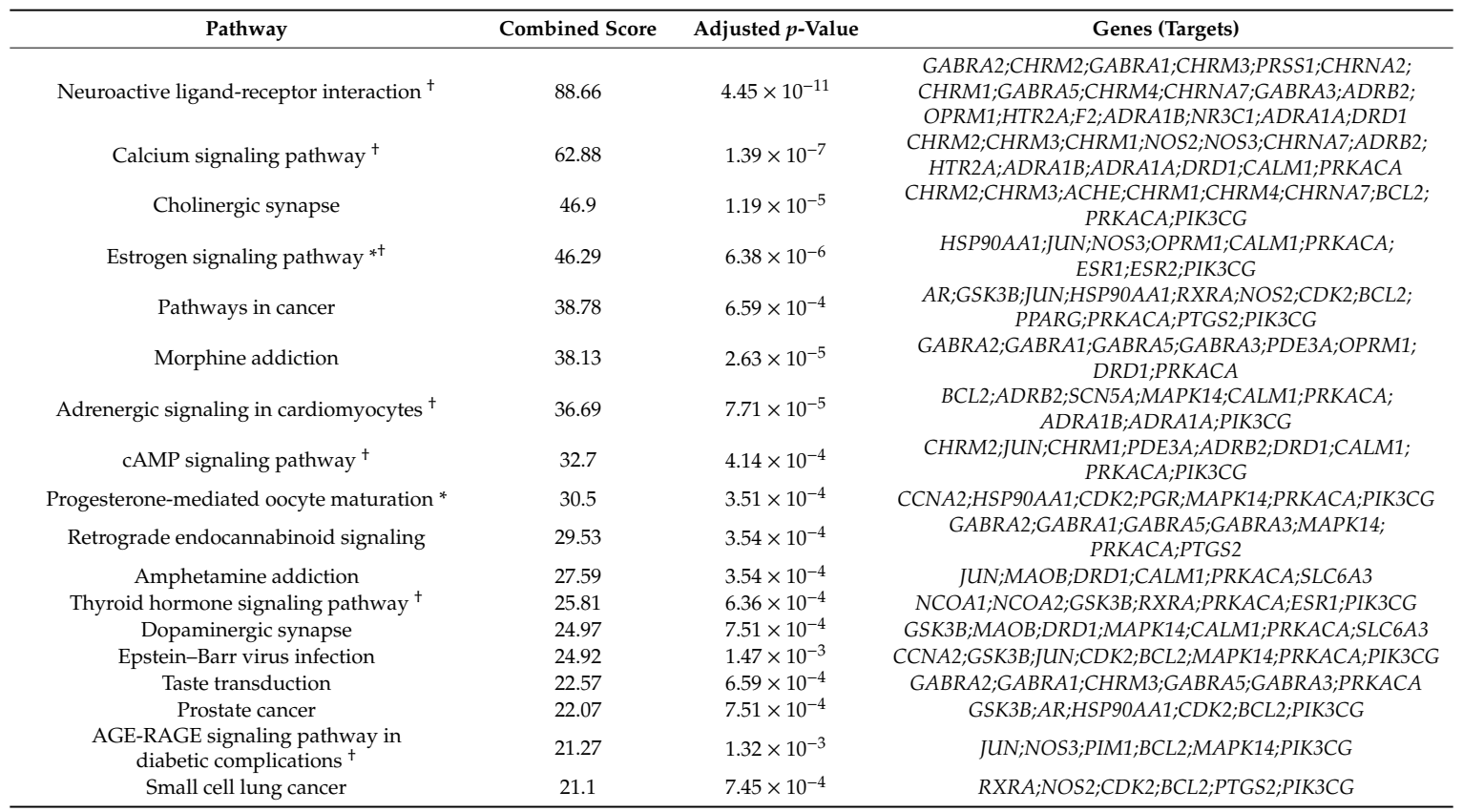


Table 4. Cont.

\begin{tabular}{|c|c|c|c|}
\hline Pathway & Combined Score & Adjusted $p$-Value & Genes (Targets) \\
\hline PI3K-Akt signaling pathway ${ }^{\dagger}$ & 20.52 & $7.80 \times 10^{-3}$ & $\begin{array}{c}\text { CHRM2;GSK3B;HSP90AA1;CHRM1;RXRA;NOS3;CDK2; } \\
\text { BCL2;PIK3CG }\end{array}$ \\
\hline Salivary secretion $^{+}$ & 19.81 & $7.51 \times 10^{-4}$ & CHRM3;ADRB2;CALM1;ADRA1B;PRKACA;ADRA1A \\
\hline Nicotine addiction & 18.71 & $3.80 \times 10^{-4}$ & GABRA2;GABRA1;GABRA5;CHRNA7;GABRA3 \\
\hline Serotonergic synapse & 16.7 & $2.05 \times 10^{-3}$ & MAOB;HTR2A;PRKACA;PTGS2;SLC6A4;PTGS1 \\
\hline Neurotrophin signaling pathway & 16.19 & $2.59 \times 10^{-3}$ & GSK3B;JUN;BCL2;MAPK14;CALM1;PIK3CG \\
\hline Regulation of lipolysis in adipocytes ${ }^{+}$ & 15.31 & $8.42 \times 10^{-4}$ & ADRB2;PTGS2;PRKACA;PIK3CG;PTGS1 \\
\hline Prolactin signaling pathway * + & 14.06 & $2.12 \times 10^{-3}$ & GSK3B;MAPK14;ESR1;ESR2;PIK3CG \\
\hline Inflammatory mediator regulation of TRP channels & 13.19 & $6.91 \times 10^{-3}$ & HTR2A;MAPK14;CALM1;PRKACA;PIK3CG \\
\hline Alcoholism & 12.61 & $1.34 \times 10^{-2}$ & MAOB;PKIA;DRD1;CALM1;PRKACA;SLC6A3 \\
\hline
\end{tabular}

+ indicates that the pathway is related to the overlapping genes between the predicted targets of PL and menopause-related genes retrieved from the Entrez Gene database; * indicates that the pathway is related to sex hormones.

\subsubsection{Construction of the Compound-Target Network of Pueraria lobata}

We constructed and visualized a bipartite compound-target network of PL to understand their interactions at the systems level. The network consisted of 65 nodes corresponding to the potential bioactive compounds or their targets and 109 edges indicating interactions between compounds and targets (Figure 6). Among the targets of formononetin, 3'-methoxydaidzein, beta-sitosterol, and daidzein-4,7-diglucoside, 15 targets were found to be related to female sex hormone-related pathways: PIK3CG, PRKACA, JUN, NOS3, CALM1, MAPK14, ESR1, ESR2, HSP90AA1, PTGS2, CCNA2, CDK2, PGR, OPRM1, and GSK3B. The numbers of related targets for the estrogen signaling pathway, progesterone-mediated oocyte maturation, oxytocin signaling pathway, and prolactin signaling pathway were $9,7,6$, and 5, respectively.

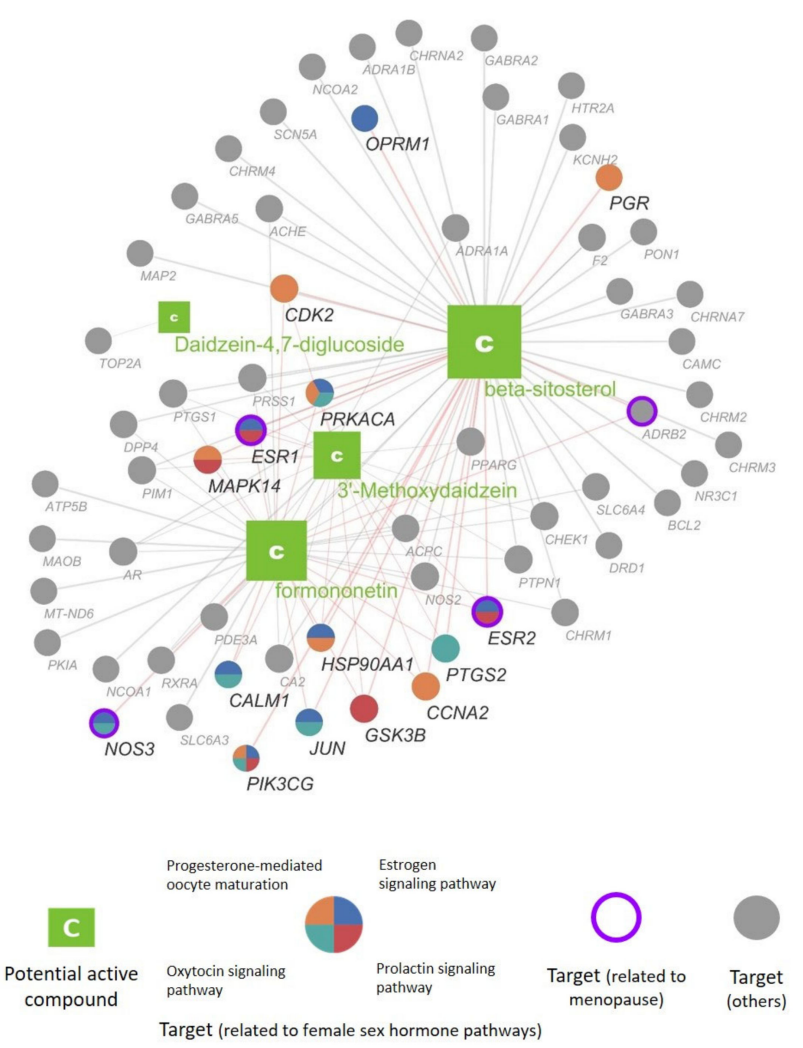

Figure 6. Compound-target network of PL. Each node represents a compound or target (explained in the box), and the edge indicates the interaction between the compound and the target. The size of each node is proportional to its degree (the number of edges). Genes associated with menopause or female sex hormone-related pathways are highlighted with different colors in the network. 


\section{Discussion}

In this study, we investigated the multiple effects and underlying mechanisms of PL on menopause-related metabolic diseases. The administration of PL significantly improved the serum lipids, GOT, and GPT in OVX rats, implying that PL has potential as a therapeutic agent for menopause-related chronic diseases. Suppressed PPAR- $\gamma$ expression in the OVX rats was significantly attenuated after treatment with PL. Additionally, network pharmacological analysis has led to the identification of bioactive compounds and their potential pathways, which suggests that the mechanism of PL might be exerted via multiple pathways related to female sex hormones. We expect that PL would have preventive effects on dyslipidemia and CVD after menopause.

In menopausal women, estrogen deficiency increases the risk of hyperlipidemia, CVD, and liver disease. This is related to the role of estrogen in lipid metabolism in which it inhibits serum low density lipoprotein (LDL) cholesterol, increases HDL cholesterol production, and inhibits homocysteine production and vascular function. Activation of estrogen receptors improves peripheral energy and glucose homeostasis in multiple ways. It prevents liver steatosis, suppresses hepatic glucose production, and improves insulin sensitivity [1]. It enhances lipid oxidation, insulin-sensitive glucose transporter GLUT4 expression, and insulin sensitivity in skeletal muscle [29].

Previous studies have shown elevated GOT, GPT, hepatocyte hypertrophy, and fat changes in the liver in OVX rats $[30,31]$. This study also supports the possibility that PL can be used for the treatment and prevention of menopause-related metabolic disorders. The levels of total cholesterol and triglyceride in the OVX model were significantly improved by PL administration, implying the possibility that PL could be used for the prevention of postmenopausal CVD and dyslipidemia. In addition, GPT and GOT were significantly decreased, confirming that PL showed a liver protective effect in the OVX model.

The PPAR- $\gamma$, analyzed as a common target of beta-sitosterol, formononetin, and $3^{\prime}$-methoxydaidzein, is a nuclear receptor whose genetic variants result in altered insulin sensitivity and lipid storage [32]. It is expressed at low levels in skeletal muscle, where it protects against adiposity and insulin resistance. The PPAR- $\gamma$ action directly promotes myocellular storage of energy by increasing fatty acid uptake and esterification while simultaneously enhancing insulin signaling and glycogen formation [33]. Muscle-specific PPAR- $\gamma$ knockout (Mu PPAR- $\gamma$ KO) mouse studies have revealed the additional metabolic importance of skeletal muscle PPAR- $\gamma$ as these mice exhibit elevated serum lipids and excess weight gain [34,35]. We observed that the expression of PPAR- $\gamma$ was significantly increased in all PL administration groups regardless of dose. Therefore, our experimental results and network pharmacological analysis consistently suggest PPAR- $\gamma$ as a candidate target of PL in lipid metabolism.

Using network pharmacological analyses, we identified four potential bioactive compounds of PL satisfying the criteria of OB and DL: formononetin, 3'-methoxydaidzein, beta-sitosterol, and daidzein-4,7-diglucoside. Formononetin has been reported to have estrogenic effects and has also been shown to improve antioxidant defense mechanisms through the enzymatic and nonenzymatic systems of tissues and to inhibit lipid peroxidation by eliminating free radicals [36]. Beta-sitosterol is known to be effective in hypercholesterolemia, heart disease, immune system regulation, and cancer prevention [37]. In particular, LDL cholesterol has been shown to act in a competitive manner to inhibit cholesterol absorption in the body, thereby reducing blood cholesterol and $\beta$-lipoprotein levels [38]. Additionally, it promoted estrogen-responsive breast cancer cell proliferation, acting similarly to estrogen [39]. 3'-Methoxydaidzein is reported to have estrogenic activity affecting breast cancer cells [40]. Daidzein-4,7-diglucoside is regarded to act as a phytoestrogen [41].

Among the 61 potential targets of PL, we retrieved four genes that are related to menopause: ESR1, ESR2, NOS3, and ADRB2. The accordance rate (four matched targets of 61 predicted targets) was higher than the chance level ( $p$-value $<0.001$, hypergeometric test), supporting the reliability of the predicted results. The ESR1 and ESR2, which are involved in the estrogen signaling pathway, are highly related to menopause-related metabolic diseases [42]. A previous study has shown that ESR1 and NOS3 predict postmenopausal CVD-related endothelial responses and can be used to predict 
insulin resistance and type 2 diabetes risk [43]. The $A D R B 2$ is known to be an obesity-related gene and is associated with disturbances in $\beta$-adrenoceptor-mediated lipolysis and fat oxidation [44], so it may affect lipid metabolism after menopause.

\section{Conclusions}

We found that PL improved various indicators associated with lipid metabolism in the postmenopausal animal model and that it simultaneously acts on several targets related to menopause-related metabolic diseases. Our results suggested that the PL targets could change serum lipid levels and liver function indicators in the OVX model by affecting multiple pathways, including the estrogen-related pathway. We expect the network pharmacological method, which systematically analyzes the action of drugs, will play a role in presenting a new direction in research design and interpretation of the underlying mechanism of drugs in the future.

Author Contributions: Conceptualization, K.S.K.; H.L.L. and J.-E.Y.; methodology, J.H.O.; W.-Y.L. and C.-E.K.; software, J.H.O.; validation, K.S.K.; C.-E.K. and J.-E.Y.; formal analysis, J.H.O. and T.A.T.; investigation, J.H.O.; S.-E.B.; J.Y.B. and D.H.P.; resources, J.Y.B.; T.A.T.; D.H.P. and K.S.K.; writing-original draft, J.H.O.; S.-E.B.; W.-Y.L. and J.-E.Y.; writing-review \& editing, H.L.L.; visualization, J.H.O.; W.-Y.L. and D.H.P.; supervision, K.S.K.; C.-E.K. and J.-E.Y.; project administration, K.S.K. and C.-E.K.; funding acquisition, J.-E.Y.

Funding: This work was supported by the Young Researchers Program through the National Research Foundation of South Korea (NRF-2017R1C1B1011984). This research was supported by the Daejeon University Research Grants, South Korea (2017).

Conflicts of Interest: The authors declare no conflict of interest.

\section{References}

1. Mauvais-Jarvis, F. Estrogen and androgen receptors: Regulators of fuel homeostasis and emerging targets for diabetes and obesity. Trends Endocrinol. Metab. 2011, 22, 24-33. [CrossRef]

2. Zapantis, G.; Santoro, N. The menopausal transition: Characteristics and management. Best Pract. Res. Clin. Endocrinol. Metab. 2003, 17, 33-52. [CrossRef]

3. Hallajzadeh, J.; Khoramdad, M.; Izadi, N.; Karamzad, N.; Almasi-Hashiani, A.; Ayubi, E.; Qorbani, M.; Pakzad, R.; Hasanzadeh, A.; Sullman, M.J.M.; et al. Metabolic syndrome and its components in premenopausal and postmenopausal women: A comprehensive systematic review and meta-analysis on observational studies. Menopause 2018, 25, 1155-1164. [CrossRef]

4. Stefanska, A.; Bergmann, K.; Sypniewska, G. Metabolic Syndrome and Menopause: Pathophysiology, Clinical and Diagnostic Significance. In Advances in Clinical Chemistry; Academic Press: Cambridge, MA, USA, 2015; ISBN 9780128033142.

5. Park, Y.W.; Zhu, S.; Palaniappan, L.; Heshka, S.; Carnethon, M.R.; Heymsfield, S.B. The metabolic syndrome: Prevalence and associated risk factor findings in the US population from the Third National Health and Nutrition Examination Survey, 1988-1994. Arch. Intern. Med. 2003, 163, 427-436. [CrossRef]

6. Jouyandeh, Z.; Nayebzadeh, F.; Qorbani, M.; Asadi, M. Metabolic syndrome and menopause. J. Diabetes Metab. Disord. 2013, 12, 1. [CrossRef]

7. Chang, W.-C.; Wang, J.-H.; Ding, D.-C. Hormone therapy in postmenopausal women associated with risk of stroke and venous thromboembolism: A population-based cohort study in Taiwan. Menopause 2019, 26, 197-202. [CrossRef]

8. Trimarco, V.; Rozza, F.; Izzo, R.; de Leo, V.; Cappelli, V.; Riccardi, C.; di Carlo, C. Effects of a new combination of nutraceuticals on postmenopausal symptoms and metabolic profile: A crossover, randomized, double-blind trial. Int. J. Womens. Health 2016, 8, 581-587. [CrossRef]

9. Jun, J.H.; Lee, H.W.; Zhang, J.; Yang, F.; Lee, M.S. Herbal medicine (Danggui Liuhuang decoction) for managing menopausal symptoms: A protocol of systematic review of randomized clinical trials. Medicine (Baltimore) 2018, 97, e9735. [CrossRef]

10. Kargozar, R.; Azizi, H.; Salari, R. A review of effective herbal medicines in controlling menopausal symptoms. Electron. Physician 2017, 9, 5826-5833. [CrossRef] 
11. Franco, O.H.; Chowdhury, R.; Troup, J.; Voortman, T.; Kunutsor, S.; Kavousi, M.; Oliver-Williams, C.; Muka, T. Use of plant-based therapies and menopausal symptoms: A systematic review and meta-analysis. JAMA J. Am. Med. Assoc. 2016, 315, 2554-2563. [CrossRef]

12. Liu, Z.M.; Ho, S.C.; Chen, Y.M.; Ho, S.; To, K.; Tomlinson, B.; Woo, J. Whole soy, but not purified daidzein, had a favorable effect on improvement of cardiovascular risks: A 6-month randomized, double-blind, and placebo-controlled trial in equol-producing postmenopausal women. Mol. Nutr. Food Res. 2014, 58, 709-717. [CrossRef] [PubMed]

13. Park, I.S.; Lee, H.W.; Ryuk, J.A.; Ko, B.S. Effects of an aqueous extract of dangguijagyagsan on serum lipid levels and blood flow improvement in ovariectomized rats. Evid. Based Complement. Altern. Med. 2014, 2014, 497836. [CrossRef] [PubMed]

14. Go, H.; Ryuk, J.A.; Lee, H.W.; Ko, B.S. Palmiwon attenuates hepatic lipid accumulation and hyperlipidemia in a menopausal rat model. Menopause 2015, 4, 42-43. [CrossRef] [PubMed]

15. Wong, K.H.; Li, G.Q.; Li, K.M.; Razmovski-Naumovski, V.; Chan, K. Kudzu root: Traditional uses and potential medicinal benefits in diabetes and cardiovascular diseases. J. Ethnopharmacol. 2011, 134, 584-607. [CrossRef]

16. Lee, M.-K.; Cho, S.-Y.; Jang, J.-Y.; Choi, M.-S.; Jeon, S.-M.; Jang, M.K.; Kim, M.-J.; Park, Y.B. Effects of Puerariae Flos and Puerariae Radix Extracts on Antioxidant Enzymes in Ethanol-Treated Rats. Am. J. Chin. Med. 2003, 29, 343-354. [CrossRef]

17. Zhang, R.; Hu, Y.; Yuan, J.; Wu, D. Effects of Puerariae radix extract on the increasing intestinal permeability in rat with alcohol-induced liver injury. J. Ethnopharmacol. 2009, 126, 207-214. [CrossRef]

18. Luo, Y.; Zheng, S.; Ding, Y.; Dai, Y.; Zhou, Y.; Xiang, R.; Bay-Jensen, A.C.; Karsdal, M.A.; Qvist, P.; Zheng, Q. Preventive effects of kudzu root on bone loss and cartilage degradation in ovariectomized rat. Am. J. Transl. Res. 2017, 9, 5180.

19. Jung, H.W.; Kang, A.N.; Kang, S.Y.; Park, Y.K.; Song, M.Y. The root extract of Pueraria lobata and its main compound, puerarin, prevent obesity by increasing the energy metabolism in skeletal muscle. Nutrients 2017, 9, 33. [CrossRef]

20. Prasain, J.K.; Jones, K.; Kirk, M.; Wilson, L.; Smith-Johnson, M.; Weaver, C.; Barnes, S. Profiling and quantification of isoflavonoids in kudzu dietary supplements by high-performance liquid chromatography and electrospray ionization tandem mass spectrometry. J. Agric. Food Chem. 2003, 51, 4213-4218. [CrossRef]

21. Ahn, S.Y.; Jo, M.S.; Lee, D.; Baek, S.E.; Baek, J.; Yu, J.S.; Jo, J.; Yun, H.; Kang, K.S.; Yoo, J.E.; et al. Dual effects of isoflavonoids from Pueraria lobata roots on estrogenic activity and anti-proliferation of MCF-7 human breast carcinoma cells. Bioorg. Chem. 2019, 83, 135-144. [CrossRef]

22. Liu, Z.H.; Sun, X.B. Network pharmacology: New opportunity for the modernization of traditional Chinese medicine. Yaoxue Xuebao 2012, 47, 696-703.

23. Ru, J.; Li, P.; Wang, J.; Zhou, W.; Li, B.; Huang, C.; Li, P.; Guo, Z.; Tao, W.; Yang, Y.; et al. TCMSP: A database of systems pharmacology for drug discovery from herbal medicines. J. Cheminform. 2014, 6, 13. [CrossRef] [PubMed]

24. Xu, X.; Zhang, W.; Huang, C.; Li, Y.; Yu, H.; Wang, Y.; Duan, J.; Ling, Y. A novel chemometric method for the prediction of human oral bioavailability. Int. J. Mol. Sci. 2012, 13, 6964-6982. [CrossRef] [PubMed]

25. Kanehisa, M.; Furumichi, M.; Tanabe, M.; Sato, Y.; Morishima, K. KEGG: New perspectives on genomes, pathways, diseases and drugs. Nucleic Acids Res. 2017, 45, D353-D361. [CrossRef]

26. Maglott, D.; Ostell, J.; Pruitt, K.D.; Tatusova, T. Entrez gene: Gene-centered information at NCBI. Nucleic Acids Res. 2011, 39, D52-D57. [CrossRef]

27. Shannon, P.; Markiel, A.; Ozier, O.; Baliga, N.S.; Wang, J.T.; Ramage, D.; Amin, N.; Schwikowski, B.; Ideker, T. Cytoscape: A software environment for integrated models of biomolecular interaction networks. Genome Res. 2003, 13, 2498-2504. [CrossRef]

28. Yu, H.; Chen, J.; Xu, X.; Li, Y.; Zhao, H.; Fang, Y.; Li, X.; Zhou, W.; Wang, W.; Wang, Y. A systematic prediction of multiple drug-target interactions from chemical, genomic, and pharmacological data. PLoS ONE 2012, 7, e37608. [CrossRef]

29. Barros, R.P.A.; Machado, U.F.; Warner, M.; Gustafsson, J.-A. Muscle GLUT4 regulation by estrogen receptors ERbeta and ER. Proc. Natl. Acad. Sci. USA 2006, 103, 1605-1608. [CrossRef]

30. Zhang, L.; Zhou, M.; Fang, G.; Tang, Y.; Chen, Z.; Liu, X. Hypocholesterolemic effect of capsaicinoids by increased bile acids excretion in ovariectomized rats. Mol. Nutr. Food Res. 2013, 57, 1080-1088. [CrossRef] 
31. Katsuta, O.; Hiratsuka, H.; Matsumoto, J.; Tsuchitani, M.; Umemura, T.; Marumo, F. Ovariectomy enhances cadmium-induced nephrotoxicity and hepatotoxicity in rats. Toxicol. Appl. Pharmacol. 1993, 119, 267-274. [CrossRef]

32. Gouda, H.N.; Sagoo, G.S.; Harding, A.H.; Yates, J.; Sandhu, M.S.; Higgins, J.P.T. The association between the peroxisome proliferator-activated receptor- $\gamma 2$ (PPARG2) Pro12Ala gene variant and type 2 diabetes mellitus: A HuGE review and meta-analysis. Am. J. Epidemiol. 2010, 171, 645-655. [CrossRef] [PubMed]

33. Hu, S.; Yao, J.; Howe, A.A.; Menke, B.M.; Sivitz, W.I.; Spector, A.A.; Norris, A.W. Peroxisome Proliferator-Activated Receptor $\gamma$ Decouples Fatty Acid Uptake from Lipid Inhibition of Insulin Signaling in Skeletal Muscle. Mol. Endocrinol. 2012, 26, 977-988. [CrossRef] [PubMed]

34. Norris, A.W.; Chen, L.; Fisher, S.J.; Szanto, I.; Ristow, M.; Jozsi, A.C.; Hirshman, M.F.; Rosen, E.D.; Goodyear, L.J.; Gonzalez, F.J.; et al. Muscle-specific PPAR $\gamma$-deficient mice develop increased adiposity and insulin resistance but respond to thiazolidinediones. J. Clin. Investig. 2003, 112, 608-618. [CrossRef] [PubMed]

35. Hevener, A.L.; He, W.; Barak, Y.; Le, J.; Bandyopadhyay, G.; Olson, P.; Wilkes, J.; Evans, R.M.; Olefsky, J. Muscle-specific Pparg deletion causes insulin resistance. Nat. Med. 2003, 9, 1491-1497. [CrossRef] [PubMed]

36. Mu, H.; Bai, Y.H.; Wang, S.T.; Zhu, Z.M.; Zhang, Y.W. Research on antioxidant effects and estrogenic effect of formononetin from Trifolium pratense (red clover). Phytomedicine 2009, 16, 314-319. [CrossRef] [PubMed]

37. Saeidnia, S. The Story of Beta-sitosterol-A Review. Eur. J. Med. Plants 2014, 4, 590. [CrossRef]

38. Farquhar, J.W.; Smith, R.E.; Dempsey, M.E. The Effect of Beta Sitosterol on the Serum Lipids of Young Men with Arteriosclerotic Heart Disease. Circulation 1956, 14, 77-82. [CrossRef]

39. Ju, Y.H.; Helferich, W.G.; Clausen, L.M.; Allred, K.F.; Almada, A.L. $\beta$-Sitosterol, $\beta$-Sitosterol Glucoside, and a Mixture of $\beta$-Sitosterol and $\beta$-Sitosterol Glucoside Modulate the Growth of Estrogen-Responsive Breast Cancer Cells in Vitro and in Ovariectomized Athymic Mice. J. Nutr. 2004, 134, 1145-1151. [CrossRef]

40. Umehara, K.; Nemoto, K.; Matsushita, A.; Terada, E.; Monthakantirat, O.; De-Eknamkul, W.; Miyase, T.; Warashina, T.; Degawa, M.; Noguchi, H. Flavonoids from the heartwood of the Thai medicinal plant Dalbergia parviflora and their effects on estrogenic-responsive human breast cancer cells. J. Nat. Prod. 2009, 72, 2163-2168. [CrossRef]

41. Casanova, M.; You, L.; Gaido, K.W.; Archibeque-Engle, S.; Janszen, D.B.; Heck, H.D.A. Developmental effects of dietary phytoestrogens in Sprague-Dawley rats and interactions of genistein and daidzein with rat estrogen receptors $\alpha$ and $\beta$ in vitro. Toxicol. Sci. 1999, 51, 236-244. [CrossRef]

42. Kuźbicka, K.; Rachoń, D.; Woziwodzka, A.; Rybicka, M.; Bielawski, K.P. Associations of ESR1 and ESR2 gene polymorphisms with metabolic syndrome and its components in postmenopausal women. Maturitas 2018, 115, 97-102. [CrossRef] [PubMed]

43. Clapauch, R.; Mourão, A.F.; Mecenas, A.S.; Maranhão, P.A.; Rossini, A.; Bouskela, E. Endothelial function and insulin resistance in early postmenopausal women with cardiovascular risk factors: Importance of ESR1 and NOS3 polymorphisms. PLoS ONE 2014, 9, e103444. [CrossRef] [PubMed]

44. Jocken, J.W.E.; Blaak, E.E.; Schiffelers, S.; Arner, P.; Van Baak, M.A.; Saris, W.H.M. Association of a beta-2 adrenoceptor (ADRB2) gene variant with a blunted in vivo lipolysis and fat oxidation. Int. J. Obes. 2007, 31, 813. [CrossRef] [PubMed]

(C) 2019 by the authors. Licensee MDPI, Basel, Switzerland. This article is an open access article distributed under the terms and conditions of the Creative Commons Attribution (CC BY) license (http://creativecommons.org/licenses/by/4.0/). 